\title{
INFLUÊNCIA DO TAMANHO E FORMA DA UNIDADE AMOSTRAL SOBRE A ESTRUTURA DE DEPENDÊNCIA ESPACIAL EM QUATRO FORMAÇÕES FLORESTAIS DO ESTADO DE SÃO PAULO
}

\author{
Melissa Oda-Souza ${ }^{1}$, João Luís Ferreira Batista ${ }^{2}$, Paulo Justiniano Ribeiro $\mathrm{Jr}^{3}$, Ricardo Ribeiro Rodrigues ${ }^{4}$ \\ ${ }^{1}$ Eng ${ }^{\mathrm{a}}$ Agrônoma, Dr ${ }^{\mathrm{a}}$, ESALQ/USP, Piracicaba, SP, Brasil - melissa.oda @ gmail.com \\ ${ }^{2}$ Eng. Florestal, Ph.D., Depto. de Ciências Florestais, ESALQ/USP, Piracicaba, SP, Brasil - parsival@usp.br \\ ${ }^{3}$ Eng. Agrônomo,Dr., Laboratório de Estatística e Geoinformação, UFPR, Curitiba, PR, Brasil - paulojus@ufpr.br \\ ${ }^{4}$ Biológo, Dr., Depto. de Ciências Biológicas, ESALQ/USP, Piracicaba, SP, Brasil - rrr@esalq.usp.br \\ Recebido para publicação: 20/06/2009 - Aceito para publicação: 23/03/2010
}

\begin{abstract}
Resumo
O tamanho e forma da unidade amostral tem grande influência na percepção da autocorrelação espacial. Dessa forma, a escolha de uma escala adequada para o estudo de processos espaciais é extremamente importante, pois a dinâmica espacial pode ser considerada significante, insignificante ou inoperante a depender da escala utilizada ou, ainda, as relações entre as variáveis podem ser obscurecidas ou distorcidas. O objetivo deste trabalho foi estudar, por meio de técnicas geoestatísticas baseadas em modelos, a estrutura de continuidade espacial, analisando a influência do tamanho e forma das unidades amostrais. Para este estudo, quatro parcelas permanentes de $320 \times 320 \mathrm{~m}$ foram divididas em grades utilizando parcelas quadradas de $5 \times 5 \mathrm{~m}, 10 \times 10 \mathrm{~m}$ e $20 \times 20 \mathrm{~m}$, e retangulares $10 \times 20 \mathrm{~m}, 10 \times 30 \mathrm{~m}, 10 \times 40 \mathrm{~m}, 10 \times 50 \mathrm{~m}$ e $5 \times 80 \mathrm{~m}$ sendo determinadas a área basal e biomassa para cada parcela. É clara a influência do tamanho e forma da parcela sobre a percepção da estrutura de continuidade espacial. No entanto, as parcelas de formas retangulares de $10 \mathrm{x} 40 \mathrm{~m}$ foram capazes de captar a variabilidade das formações e parcelas de 20 × 20 m descreveram melhor a estrutura espacial.
\end{abstract}

Palavras-chave: Parcela permanente; formações florestais; geoestatística baseada em modelos; critério de informação de Akaike.

\begin{abstract}
Influence of sample unit size and shape on spatial structure dependence in four forest formations in the State of São Paulo, Brazil. The size and shape of sampling units have a large influence on the perception of spatial autocorrelation. Thus, the choice of an appropriate scale for the study of spatial processes is extremely important, because the spatial dynamics can be considered as significant, insignificant or irrelevant depending on the scale used, or the relationship between variables may be obscured or distorted. The aim of this work was to study the structure of spatial continuity, by modelbased geostatistical,analyzing the influence of sample unit size and shape. For this study, four permanent plots of $320 \times 320 \mathrm{~m}$ were divided into grids using square plots of $5 \times 5 \mathrm{~m}, 10 \times 10 \mathrm{~m}$ and $20 \times 20 \mathrm{~m}$, and rectangular plots of $10 \times 20 \mathrm{~m}, 10 \times 30 \mathrm{~m}, 10 \times 40 \mathrm{~m}, 10 \times 50 \mathrm{~m}$ and $5 \times 80 \mathrm{~m}$. Basal area and biomass were determined for each plot. The influence of size and shape of the plot on the perception of the structure of spatial continuity was clear. However, the rectangular plots of $10 \mathrm{x} 40 \mathrm{~m}$ were able to identify the variability of the forest, and the plots of $20 \times 20 \mathrm{~m}$ were the best to describe the structure of spatial continuity.
\end{abstract}

Keywords: Permanent plot; forest formations; model-based geostatistics; Akaike information criterion.

\section{INTRODUÇÃO}

Consideráveis esforços têm sido dedicados ao desenvolvimento de delineamentos amostrais e técnicas estatísticas para detectar e quantificar a estrutura de continuidade espacial em estudos ecológicos (ROSSI et al., 1992; DUTILLEUL, 1993; GOSLEE, 2006; DORMANN, 2007). No entanto ainda são poucos os estudos (BELLEHUMEUR et al., 1997; FORTIN, 1999; ROSSI; NUUTINEN, 2004) 
realizados para verificar a influência da mudança de escala na percepção da autocorrelação espacial.

Segundo Gotway; Young (2002), a escolha de uma escala adequada para o estudo de processos espaciais é extremamente importante. A dinâmica espacial de um processo pode ser considerada significante, insignificante ou inoperante, a depender da escala utilizada, ou, ainda, as relações entre as variáveis podem ser obscurecidas ou distorcidas.

O conceito de escala pode ser definido de diferentes formas nas diversas áreas do conhecimento, mas em levantamentos ecológicos refere-se principalmente aos componentes do delineamento amostral (HE et al., 2002). Bellehumeur; Legendre (1998) e Dungan et al. (2002) descrevem três componentes amostrais como essenciais para a percepção da estrutura espacial: 1) tamanho da unidade amostral, que é a superfície ou volume em que a variável de interesse será mensurada; 2) forma da unidade amostral, sendo as mais comuns as quadradas, retangulares e circulares; e 3) distância entre unidades amostrais. Os dois primeiros componentes, tamanho e forma, têm influência direta sobre a variável mensurada, pois mudanças no tamanho e forma da unidade amostral criam uma nova variável, induzindo a alterações na variância e na estrutura de continuidade espacial. O último componente, distância entre as unidades amostrais, tem influência sobre a vizinhança.

Nos últimos anos, muitas técnicas, como o autocorrelograma (LEGENDRE; FORTIN, 1989), covariograma (SATAKE; IWASA, 2002), coeficiente de autocorrelação espacial de Moran's I (FORTIN, 1999), análise fractal (CHEN et al., 2002), geoestatísticas baseadas em variogramas (ISAAKS; SRISVASTAVA, 1989) e geoestatística baseada em modelo (DIGGLE et al., 1998; DIGGLE; RIBEIRO JR., 2007), foram propostas para o estudo da continuidade espacial. Técnicas geoestatísticas têm sido aplicadas em estudos ecológicos e florestais (HERNANDEZ-STEFANONI, 2005; HOLMES et al., 2006; SALES et al., 2007).

Tradicionalmente, das técnicas geoestatísticas, as baseadas em variogramas têm sido as mais utilizadas para a modelagem da estrutura de continuidade espacial, mas com a desvantagem de não assumir explicitamente nenhuma distribuição (PLONER; DUTTER, 2000) e de serem obtidos resultados muitas vezes imprecisos, em função dos diferentes números de pares que geram cada ponto do variograma e por cada ponto entrar várias vezes no cálculo das distâncias (OLIVEIRA, 2003).

A geoestatística baseada em modelos, termo cunhado por Diggle et al. (1998), descreve problemas geoestatísticos fundamentados na aplicação de métodos estatísticos formais, com a explicitação de um modelo e métodos de inferência baseados na verossimilhança. As vantagens da explicitação de modelos associada a verossimilhança são maior eficiência na estimação de parâmetros e a possibilidade de avaliar a incerteza envolvida na estimativa da estrutura de dependência (DIGGLE; RIBEIRO JR., 2007). Ao contrário da geoestatística baseada em variogramas, essa incerteza pode ser facilmente integrada na previsão dos modelos (PLONER; DUTTER, 2000).

Considerando que as estruturas de dependência espacial variam entre as formações florestais e que o tamanho e a forma da parcela influenciam na percepção dessa estrutura, diferentes modelos geoestatísticos podem se ajustar a diferentes formações, ou mesmo um único modelo ajustado pode apresentar diferenças marcantes para as estimativas dos parâmetros.

Assim, o objetivo deste trabalho foi estudar, por meio de técnicas geoestatísticas baseadas em modelos, a estrutura de continuidade espacial em quatro formações florestais, analisando a influência do tamanho e forma das unidades amostrais sobre a estrutura espacial.

\section{MATERIAL E MÉTODOS}

\section{Área de estudo}

Os dados utilizados neste trabalho são provenientes do Projeto "Diversidade, dinâmica e conservação em florestas do Estado de São Paulo: 40 ha de parcelas permanentes", do Programa Biota, da FAPESP (RODRIGUES, 2002). Foram instaladas parcelas permanentes em quatro Unidades de Conservação do Estado de São Paulo (Figura 1A), que representam as principais formações florestais do estado: Floresta de Restinga (Parque Estadual Ilha do Cardoso), Floresta Ombrófila Densa Montana (Parque Estadual de Carlos Botelho), Floresta Estacional Semidecidual (Estação Ecológica dos Caetetus) e Cerrado lato sensu ou Savana Florestada (Estação Ecológica de Assis). Em cada Unidade de Conservação foi alocada uma parcela permanente de $320 \times 320 \mathrm{~m}$, totalizando 10,24 ha, subdividida em 256 subparcelas contíguas de $20 \times 20 \mathrm{~m}\left(400 \mathrm{~m}^{2}\right)$ (Figura 1B). Todos os indivíduos arbóreos no interior 
das parcelas com diâmetro (DAP - diâmetro à altura do peito) maior ou igual a $5 \mathrm{~cm}$ foram mapeados, georreferenciados, medidos e identificados. Com essas informações, foram construídos mapas de todas as árvores das parcelas permanentes com suas coordenadas.
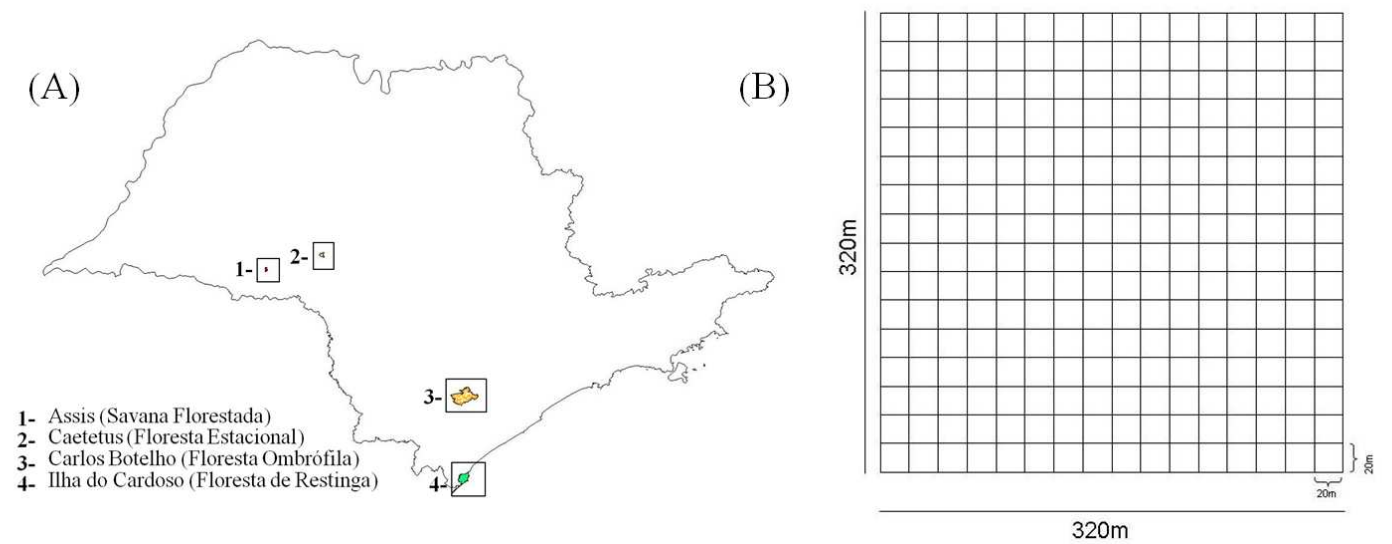

Figura 1. (A) Localização das quatro Unidades de Conservação no estado de São Paulo; (B) Parcela permanente subdividida em 256 subparcelas contíguas de $20 \times 20 \mathrm{~m}\left(400 \mathrm{~m}^{2}\right)$.

Figure 1. (A) Location of the four Conservation Units in the state of São Paulo; (B) Permanent plot subdivided into 256 contiguous subplots of $20 \times 20 \mathrm{~m}\left(400 \mathrm{~m}^{2}\right)$.

\section{Tamanho e forma das parcelas estudadas}

As parcelas permanentes foram divididas em grades utilizando-se parcelas quadradas de $5 \times 5 \mathrm{~m}$ (4096 parcelas), $10 \times 10 \mathrm{~m}$ (1024 parcelas) e $20 \times 20 \mathrm{~m}$ (256 parcelas), e retangulares de $10 \times 20 \mathrm{~m}$ (512 parcelas), 10 × $30 \mathrm{~m}$ (300 parcelas), 10 × $40 \mathrm{~m}$ ( 256 parcelas), $10 \times 50 \mathrm{~m}$ (180 parcelas) e $5 \times 80 \mathrm{~m}$ (256 parcelas). Para as duas formas (quadrada e retangular) e diferentes tamanhos $\left(25 \mathrm{~m}^{2}, 100 \mathrm{~m}^{2}\right.$, $200 \mathrm{~m}^{2}, 300 \mathrm{~m}^{2}, 400 \mathrm{~m}^{2}$ e $\left.500 \mathrm{~m}^{2}\right)$ de parcelas foram determinadas área basal $\left(\mathrm{m}^{2} . \mathrm{ha}^{-1}\right)$ e

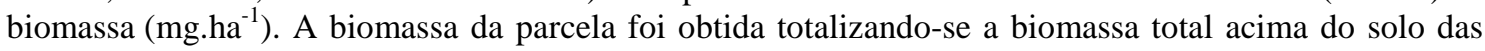
árvores individuais, utilizando-se os modelos apresentados na tabela 1.

Tabela 1. Modelos para estimativas das biomassas das árvores individuais.

Table 1. Models to estimate the biomass of individual trees.

\begin{tabular}{lcc}
\hline Formações florestais & Modelo & Fonte \\
\hline Savana Florestada e Restinga & $\mathrm{b}=\exp (-1,79532)(\mathrm{d})^{2,29744}$ & Batista, 2008 (dados não publicados) $^{1}$ \\
Estacional & $\mathrm{b}=\exp (-9,36745+2,47442(\log (\mathrm{d})))$ & Mello,2008 (dados não publicados) ${ }^{2}$ \\
Ombrófila & $\mathrm{b}=\exp (-6.80067+3,77738(\log (\mathrm{d})))$ & Burger, 2005 $^{2}$
\end{tabular}

\section{Modelo geoestatístico}

$\mathrm{O}$ formato básico para dados geoestatísticos univariados é dado por $\left(\mathrm{x}_{\mathrm{i}}, \mathrm{y}_{\mathrm{i}}\right), \mathrm{i}=1, \ldots, \mathrm{n}$, em que $\mathrm{x}_{\mathrm{i}}$ identifica a posição espacial tipicamente em um plano bidimensional e $\mathrm{y}_{\mathrm{i}}$ é uma medição escalar tomada na posição $x_{i}$. Uma característica da geoestatística é que a variável de medição $y_{i}$ pode, em princípio, estar localizada em qualquer lugar numa região de estudo (DIGGLE; RIBEIRO JR., 2007).

No contexto de delineamento de amostragem, $x_{i}$ representa a posição da unidade amostral e $y_{i}$ as variáveis de medição, como área basal e biomassa. Assumindo que os dados de área basal e biomassa $\mathrm{y}=\mathrm{y}_{1}, \ldots, \mathrm{y}_{\mathrm{n}}$ seguem o modelo linear gaussiano, o modelo geoestatístico exposto por Diggle $e$ t al. (1998) descreve a dependência espacial através de um componente de erro aleatório e espacialmente correlacionado, que é dado por $\mathrm{Y}_{\mathrm{i}}=\mu\left(\mathrm{x}_{\mathrm{i}}\right)+\mathrm{S}\left(\mathrm{x}_{\mathrm{i}}\right)+\varepsilon_{\mathrm{i}}, \mathrm{i}=1, \ldots$, $\mathrm{n}$, em que $\mathrm{Y}_{\mathrm{i}}$ é o valor observado na

${ }^{1}$ BATISTA, J. L. F. Escola Superior de Agricultura "Luiz de Queiroz", Universidade de São Paulo, Departamento de Ciências Florestais, 2008.

${ }^{2}$ MELLO, J. M. Universidade Federal de Lavras, Departamento de Ciências Florestais, 2008.

FLORESTA, Curitiba, PR, v. 40, n. 4, p. 849-860, out./dez. 2010. 
posição, $\mathrm{x}_{\mathrm{i}}$ é a posição espacial da i-ésima parcela e $\mu\left(\mathrm{x}_{\mathrm{i}}\right)$ é determinada a partir de um modelo de regressão. $\mathrm{O}$ termo $\mathrm{S}\left(\mathrm{x}_{\mathrm{i}}\right)$ denota o valor na posição de um processo estacionário gaussiano, com média zero, variância $\sigma^{2}$ e função de correlação $\rho(u ; \phi)$ com argumento dado pela distância u de separação entre observações e parametrizada por $\phi$, que descreve o grau de associação espacial. Decorre então que para um conjunto finito de observações $S \sim \operatorname{NMV}\left(0, \sigma^{2} R(\phi)\right)$, em que $R(\phi)$ é a matriz de covariância $n \times n$ com (i, j)-ésimo elemento, $\rho(u)$, sendo $u=|| x_{i}-x_{j}||$ a distância euclidiana entre $x_{i}$ e $x_{j}$.

$O$ termo $\varepsilon_{\mathrm{i}}$ denota variáveis aleatórias, independentes e normalmente distribuídas com média zero e variância $\tau^{2}$. A função de correlação $\rho(\mathrm{u})$ presente na matriz de covariância adotada foi a Matérn, $\rho(u)=\left\{2^{\kappa-1} \Gamma(\kappa)\right\}^{-1}(u / \phi)^{\kappa} K_{\kappa}(u / \phi)$, com $\kappa=0,5,1,0$ e 2,0 , em que o primeiro caso corresponde ao modelo exponencial $\rho(\mathrm{u})=\exp \{-(\mathrm{u} / \phi)\}$, e o alcance prático é aproximadamente $3 \phi, 4 \phi$ e $5,37 \phi$, respectivamente. Seguindo a terminologia geoestatística usual (ISAAKS; SRIVASTAVA, 1989), $\tau^{2}$ é o efeito pepita (nugget), $\sigma^{2}$ é o patamar (sill), e a distância a partir da origem até o patamar é chamada de alcance prático (range), que denotamos aqui por a, que é função do parâmetro $\phi$. A razão do efeito pepita $\left(\tau^{2}\right)$ com o sill total $\left(\tau^{2}+\sigma^{2}\right)$ é chamada de efeito pepita relativo $\left(\operatorname{Rel}_{\tau^{2}}\right.$ ), que representa a proporção do componente aleatório sobre a variância total. Para a transformação do modelo gaussiano, foi aplicada a família de transformação Box-Cox (BOX; COX, 1964), que é discutido no contexto de modelagem geoestatística por Christensen et al. (2001). A estimação dos parâmetros foi obtida por verossimilhança (DIGGLE; RIBEIRO JR., 2007).

\section{Seleção dos modelos}

A avaliação do desempenho dos modelos foi realizada em duas etapas. Na primeira avaliou-se a plausibilidade das diferentes funções de correlação nos modelos espaciais e, na segunda, a plausibilidade dos modelos espaciais e não-espaciais. As duas etapas foram baseadas no critério de informação de Akaike (AIC). Akaike (1974) estabeleceu uma relação entre a informação ou distância de KullbackLeibler (KULLBACK; LEIBLER. 1951), que é uma medida de discrepância relativa entre dois modelos, o modelo verdadeiro e o modelo aproximado, e a função de máxima log-verossimilhança para seleção de modelos. O AIC é definido por AIC $=-2 l+2 p$, em que 1 é o logaritmo neperiano do máximo da função de verossimilhança, calculado para os valores das estimativas dos parâmetros, e p é o número de parâmetros do modelo considerado.

O valor de AIC individual não é interpretável. Uma alternativa proposta por Burnham; Anderson (2002) para avaliar a plausibilidade dos modelos é baseada nas diferenças dos AIC $\left(\Delta_{\mathrm{i}}\right)$ e dada por $\Delta_{\mathrm{i}}=\mathrm{AIC}_{\mathrm{i}}-\mathrm{AIC}_{\mathrm{m}}$, em que $\mathrm{AIC}_{\mathrm{i}}$ é o valor de $\mathrm{AIC}$ para o modelo i e $\mathrm{AIC}_{\mathrm{m}}$ é o valor de $\mathrm{AIC}$ para o modelo m, ou seja, o modelo com menor valor de AIC. Para valores de $\Delta_{\mathrm{i}}$ maiores do que dois, o modelo i é menos plausível que o modelo $\mathrm{m}$. Os valores das diferenças dos $\operatorname{AIC}\left(\Delta_{\mathrm{i}}\right)$ são de fácil interpretação e permitem uma rápida comparação de modelos ordenados.

Especificamente para avaliar a plausibilidade dos modelos espaciais e não-espaciais, fixou-se o $\mathrm{AIC}_{\mathrm{i}}$ como o valor do AIC para o modelo não-espacial ( $\mathrm{AIC}_{\mathrm{nsp}}$ ) e o $\mathrm{AIC}_{\mathrm{m}}$ como o valor do $\mathrm{AIC}$ para o modelo espacial $\left(\mathrm{AIC}_{\mathrm{sp}}\right)$, e obteve-se $\Delta_{\mathrm{sp}}=\mathrm{AIC}_{\mathrm{nsp}}-\mathrm{AIC}_{\mathrm{sp}}$. Para valores de $\Delta_{\mathrm{sp}}$ maiores do que dois, o modelo espacial é mais plausível que o modelo não-espacial.

Antecedendo a análise geoestatística, foi realizada uma análise exploratória descritiva, para avaliar o comportamento das variáveis área basal $\left(\mathrm{m}^{2} \cdot \mathrm{ha}^{-1}\right)$ e biomassa $\left(\mathrm{mg}^{-1} \mathrm{a}^{-1}\right)$ com a mudança do tamanho e forma das parcelas nas quatro formações florestais estudadas.

Neste estudo, as análises foram realizadas utilizando-se o pacote geoR (RIBEIRO JR.; DIGGLE, 2001), do projeto R de computação estatística (R DEVELOPMENT CORE TEAM, 2008).

\section{RESULTADOS}

As estatísticas descritivas das variáveis área basal $\left(\mathrm{m}^{2} \cdot \mathrm{ha}^{-1}\right)$ e biomassa $\left(\mathrm{mg}_{\mathrm{a}} \mathrm{ha}^{-1}\right)$ para as quatro formações florestais são apresentadas nas tabelas 2 e 3 . Foi possível observar que à medida que aumenta o tamanho da parcela os valores das medidas de posição (média e mediana) ficam próximos e os desvios padrão e coeficientes de variação diminuem, sendo que parcelas de mesmo tamanho mas com formas diferentes apresentam valores próximos. 
Tabela 2. Média ( $\bar{x}$ ), mediana (Med), desvio-padrão (S) e coeficiente de variação (CV\%) da área basal $\left(\mathrm{m}^{2} \cdot \mathrm{ha}^{-1}\right)$ nas quatro formações florestais com diferentes tamanhos e formas de parcela.

Table 2. Mean ( $\bar{x})$, median (Med), standard deviation (S) and coefficient of variation (CV \%) of basal area $\left(\mathrm{m}^{2} \cdot \mathrm{ha}^{-1}\right)$ in the four forest formations with different sizes and shapes of sampling unit.

\begin{tabular}{|c|c|c|c|c|c|c|c|c|}
\hline Parcela (m) & $\bar{x}$ & Med & $\mathbf{S}$ & CV $(\%)$ & $\bar{x}$ & Med & $\mathbf{S}$ & $\mathrm{CV}(\%)$ \\
\hline & \multicolumn{4}{|c|}{ Savana } & \multicolumn{4}{|c|}{ Estacional } \\
\hline $5 \times 5$ & 22,00 & 18,72 & 15,63 & 71,05 & 25,01 & 11,87 & 44,30 & 177,13 \\
\hline $10 \times 10$ & 22,00 & 21,59 & 7,50 & 34,09 & 25,01 & 20,09 & 22,72 & 90,84 \\
\hline $20 \times 20$ & 22,00 & 21,64 & 4,22 & 19,18 & 25,01 & 22,41 & 10,75 & 42,98 \\
\hline $10 \times 20$ & 22,00 & 21,71 & 5,59 & 25,41 & 25,01 & 21,74 & 16,13 & 64,49 \\
\hline $10 \times 30$ & 22,00 & 21,80 & 4,41 & 20,05 & 24,92 & 22,56 & 12,26 & 49,20 \\
\hline $10 \times 40$ & 22,00 & 21,89 & 4,14 & 18,82 & 25,01 & 23,14 & 11,45 & 45,78 \\
\hline $10 \times 50$ & 22,00 & 21,89 & 3,76 & 17,09 & 24,92 & 23,34 & 9,99 & 40,09 \\
\hline \multirow[t]{2}{*}{$5 \times 80$} & 22,00 & 21,89 & 4,23 & 19,23 & 25,01 & 22,67 & 11,08 & 44,30 \\
\hline & \multicolumn{4}{|c|}{ Ombrófila } & \multicolumn{4}{|c|}{ Restinga } \\
\hline $5 \times 5$ & 29,04 & 10,36 & 58,63 & 201,89 & 29,12 & 18,11 & 33,67 & 115,63 \\
\hline $10 \times 10$ & 29,04 & 19,03 & 30,58 & 105,30 & 29,12 & 25,21 & 17,02 & 58,45 \\
\hline $20 \times 20$ & 29,04 & 26,84 & 18,21 & 62,71 & 29,12 & 28,11 & 8,72 & 29,95 \\
\hline $10 \times 20$ & 29,04 & 24,34 & 23,42 & 80,65 & 29,12 & 27,71 & 11,91 & 40,90 \\
\hline $10 \times 30$ & 29,33 & 25,93 & 20,11 & 68,56 & 29,00 & 27,38 & 10,24 & 35,31 \\
\hline $10 \times 40$ & 29,04 & 26,54 & 18,09 & 62,29 & 29,12 & 28,32 & 8,37 & 28,74 \\
\hline $10 \times 50$ & 29,33 & 26,93 & 17,67 & 60,25 & 29,00 & 27,62 & 8,13 & 28,03 \\
\hline $5 \times 80$ & 29,04 & 25,94 & 17,08 & 58,82 & 29,12 & 27,54 & 8,56 & 29,04 \\
\hline
\end{tabular}

Tabela 3. Média ( $\bar{x}$ ), mediana (Med), desvio-padrão (S) e coeficiente de variação (CV\%) da biomassa $\left(\mathrm{mg}^{-h^{-1}}\right)$ nas quatro formações florestais com diferentes tamanhos e formas de parcela.

Table 3. Mean $(\bar{x})$, median (Med), standard deviation (S) and coefficient of variation $(\mathrm{CV} \%)$ of biomass $\left(\mathrm{mg} \cdot \mathrm{ha}^{-1}\right)$ in the four forest formations with different sizes and shapes of sampling unit.

\begin{tabular}{|c|c|c|c|c|c|c|c|c|}
\hline Parcela (m) & $\bar{x}$ & Med & $\mathbf{S}$ & $\mathrm{CV}(\%)$ & $\bar{x}$ & Med & $\mathbf{S}$ & $\mathrm{CV}(\%)$ \\
\hline & \multicolumn{4}{|c|}{ Savana } & \multicolumn{4}{|c|}{ Estacional } \\
\hline $5 \times 5$ & 104,29 & 84,42 & 85,26 & 81,75 & 145,17 & 43,66 & 406,72 & 280,20 \\
\hline $10 \times 10$ & 104,29 & 99,78 & 40,60 & 38,93 & 145,17 & 92,71 & 209,50 & 144,31 \\
\hline $20 \times 20$ & 104,29 & 103,25 & 22,24 & 21,33 & 145,17 & 112,38 & 99,77 & 68,73 \\
\hline $10 \times 20$ & 104,29 & 103,18 & 29,88 & 28,65 & 145,17 & 108,60 & 148,14 & 102,05 \\
\hline $10 \times 30$ & 104,46 & 102,63 & 23,39 & 22,30 & 143,16 & 115,05 & 110,33 & 77,07 \\
\hline $10 \times 40$ & 104,29 & 103,14 & 21,76 & 20,86 & 145,17 & 121,51 & 104,94 & 73,30 \\
\hline $10 \times 50$ & 104,47 & 103,11 & 19,64 & 18,80 & 143,16 & 121,92 & 88,84 & 62,06 \\
\hline \multirow[t]{2}{*}{$5 \times 80$} & 104,29 & 103,67 & 22,12 & 21,21 & 145,17 & 116,21 & 102,23 & 70,42 \\
\hline & \multicolumn{4}{|c|}{ Ombrófila } & \multicolumn{4}{|c|}{ Restinga } \\
\hline $5 \times 5$ & 548,77 & 13,74 & 3202,59 & 583,59 & 162,33 & 83,94 & 224,60 & 152,96 \\
\hline $10 \times 10$ & 548,77 & 65,98 & 1580,23 & 287,96 & 162,33 & 133,24 & 113,78 & 70,09 \\
\hline $20 \times 20$ & 548,77 & 268,78 & 801,74 & 146,10 & 162,33 & 152,90 & 58,03 & 35,75 \\
\hline $10 \times 20$ & 548,77 & 175,57 & 1144,04 & 208,47 & 162,33 & 149,28 & 79,05 & 48,70 \\
\hline $10 \times 30$ & 557,64 & 227,25 & 964,28 & 172,92 & 161,64 & 149,29 & 68,94 & 42,65 \\
\hline $10 \times 40$ & 548,77 & 266,03 & 854,48 & 155,71 & 162,33 & 156,05 & 55,90 & 34,14 \\
\hline $10 \times 50$ & 557,64 & 281,47 & 807,40 & 144,79 & 161,64 & 150,72 & 54,61 & 33,78 \\
\hline $5 \times 80$ & 548,77 & 252,70 & 833,48 & 151,88 & 162,33 & 151,53 & 57,42 & 35,37 \\
\hline
\end{tabular}

Nas tabelas 4 e 5 são apresentados os valores de critério de informação de Akaike dos modelos espaciais com diferentes funções de continuidade para as variáveis de área basal e biomassa, respectivamente. Os valores de AIC sofreram maiores variações $\left(\Delta_{\mathrm{i}}>2\right)$ nas parcelas de 5 x $5 \mathrm{~m}$ para as duas variáveis na floresta Ombrófila. Para as demais parcelas os valores foram aproximadamente iguais e suas diferenças não foram maiores que dois. Assim, foi selecionada a função Matérn com $\kappa=1$ para apresentação dos próximos resultados.

FLORESTA, Curitiba, PR, v. 40, n. 4, p. 849-860, out./dez. 2010. 
Tabela 4. Critério de informação de Akaike (AIC) dos modelos espaciais com diferentes funções de continuidade (FC) das quatro formações florestais com diferentes tamanhos e formas de parcela para área basal $\left(\mathrm{m}^{2} \cdot \mathrm{ha}^{-1}\right)$.

Table 4. Akaike information criterion (AIC) for spatial models with different functions of spatial continuity (FC) for four forest formations with different sizes and shapes of sampling units for basal area $\left(\mathrm{m}^{2} \cdot \mathrm{ha}^{-1}\right)$.

\begin{tabular}{lccccc}
\hline Parcela $(\mathbf{m})$ & FC & Savana & Estacional & Ombrófila & Restinga \\
\hline $5 \times 5$ & Exponencial & 31986,32 & 28560,93 & 22386,04 & 33413,72 \\
& Matérn $(\kappa=1,0)$ & 31985,05 & 28561,02 & 22390,99 & 33413,64 \\
\multirow{5}{*}{$10 \times 10$} & Matérn $(\kappa=2,0)$ & 31984,45 & 28561,15 & 22397,62 & 33413,54 \\
& Exponencial & 6941,84 & 8450,24 & 8439,83 & 8441,76 \\
& Matérn $(\kappa=1,0)$ & 6941,46 & 8450,23 & 8439,65 & 8441,71 \\
\multirow{5}{*}{$20 \times 20$} & Matérn $(\kappa=2,0)$ & 6941,49 & 8450,23 & 8440,18 & 8441,67 \\
& Exponencial & 1454,99 & 1883,32 & 2097,96 & 1817,12 \\
& Matérn $(\kappa=1,0)$ & 1454,58 & 1883,24 & 2097,84 & 1816,98 \\
$10 \times 20$ & Matérn $(\kappa=2,0)$ & 1454,31 & 1883,16 & 2097,94 & 1816,88 \\
& Exponencial & 3205,46 & 4037,82 & 4422,20 & 3945,47 \\
& Matérn $(\kappa=1,0)$ & 3204,93 & 4037,82 & 4420,22 & 3945,47 \\
$10 \times 30$ & Matérn $(\kappa=2,0)$ & 3204,61 & 4037,82 & 4418,84 & 3945,47 \\
& Exponencial & 1733,82 & 2263,48 & 2559,61 & 2223,06 \\
& Matérn $(\kappa=1,0)$ & 1733,14 & 2263,48 & 2557,76 & 2223,05 \\
$10 \times 40$ & Matérn $(\kappa=2,0)$ & 1732,68 & 2263,48 & 2556,61 & 2223,05 \\
& Exponencial & 1445,44 & 1901,49 & 2163,81 & 1812,71 \\
& Matérn $(\kappa=1,0)$ & 1444,65 & 1901,44 & 2163,60 & 1812,71 \\
$10 \times 50$ & Matérn $(\kappa=2,0)$ & 1443,91 & 1901,40 & 2163,44 & 1812,71 \\
& Exponencial & 982,64 & 1315,24 & 1528,03 & 1251,47 \\
& Matérn $(\kappa=1,0)$ & 982,72 & 1315,24 & 1527,61 & 1251,47 \\
$5 \times 80$ & Matérn $(\kappa=2,0)$ & 982,68 & 1315,24 & 1527,53 & 1251,47 \\
& Exponencial & 1448,42 & 1879,87 & 2114,13 & 1806,68 \\
& Matérn $(\kappa=1,0)$ & 1448,46 & 1879,87 & 2114,41 & 1806,64 \\
& Matérn $(\kappa=2,0)$ & 1448,50 & 1879,87 & 2114,78 & 1806,61 \\
\hline
\end{tabular}

De forma geral, comparando as quatro formações pelos valores obtidos das diferenças dos AIC $\left(\Delta_{\mathrm{sp}}\right)$ (Tabela 6 e 7), os modelos espaciais das formações Ombrófila, seguidos da Savana Florestada, são marcadamente diferentes, mais pronunciados do que nos modelos da Estacional e da Restinga.

Considerando a plausibilidade dos modelos espaciais e não-espaciais, foi verificado pelos valores de $\Delta_{\mathrm{sp}}$ que os modelos espaciais foram mais plausíveis nas formações Savana e Ombrófila em todos os tamanhos e formas de parcela para a variável área basal (Tabela 6). Entretanto, nas parcelas $10 \times 20$ m, $10 \times 30$ m, 10 × 40 m e 10 × 50 m para Savana, e 10 × 40 m e 10 × 50 m para Ombrófila, para a variável biomassa (Tabela 7), o modelo não-espacial foi o mais plausível. Nas formações Estacional e Restinga, os valores de $\Delta_{\mathrm{sp}}$ foram maiores do que dois para as parcelas de $5 \times 5 \mathrm{~m}$ para as duas variáveis, e para biomassa na parcela de $20 \times 20 \mathrm{~m}$, respectivamente.

A proporção do componente aleatório dado pelo efeito pepita relativo $\left(\operatorname{Rel}_{\tau^{2}}\right)$ diminuiu com o aumento do tamanho da parcela e, consequentemente, aumentou a variância estruturada. Mesmo apresentando baixa proporção da variância estruturada, os modelos espaciais foram mais plausíveis para as parcelas de tamanho de $25 \mathrm{~m}^{2}$ nas formações de Savana, Estacional e Ombrófila nas variáveis avaliadas.

Pode-se observar que a estrutura de continuidade espacial da variável biomassa foi mais tênue se comparada com a da área basal. Esses resultados foram corroborados pelos valores de $\left(\Delta_{\mathrm{sp}}\right)$ e pelo alcance prático (a), que são maiores para área basal. Como exemplo, na Savana Florestada as parcelas de tamanhos $10 \times 20 \mathrm{~m}, 10 \times 30 \mathrm{~m}, 10 \times 40 \mathrm{~m}$ e $10 \times 50 \mathrm{~m}$ são estruturadas espacialmente na variável área basal, ao passo que na variável biomassa não se justifica a aplicação do modelo geoestatístico. 
Tabela 5. Critério de informação de Akaike (AIC) dos modelos espaciais com diferentes funções de continuidade (FC) das quatro formações florestais com diferentes tamanhos e formas de parcela para biomassa $\left(\mathrm{mg}^{-h^{-1}}{ }^{-1}\right)$.

Table 5. Akaike information criterion (AIC) for spatial models with different functions of spatial continuity (FC) for four forest formations with different sizes and shapes of sampling units for

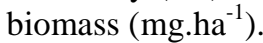

\begin{tabular}{lccccc}
\hline Parcela $(\mathbf{m})$ & FC & Savana & Estacional & Ombrófila & Restinga \\
\hline $5 \times 5$ & Exponencial & 45113,92 & 39961,51 & 29785,46 & 46620,03 \\
& Matérn $(\kappa=1,0)$ & 45112,92 & 39961,60 & 29788,58 & 46619,96 \\
$10 \times 10$ & Matérn $(\kappa=2,0)$ & 45112,51 & 39961,70 & 29793,02 & 46622,44 \\
& Exponencial & 10350,81 & 12136,20 & 12859,19 & 12142,86 \\
& Matérn $(\kappa=1,0)$ & 10350,52 & 12136,20 & 12859,10 & 12142,83 \\
$20 \times 20$ & Matérn $(\kappa=2,0)$ & 10350,51 & 12136,20 & 12859,52 & 12142,80 \\
& Exponencial & 2312,22 & 2916,76 & 3666,42 & 2773,29 \\
& Matérn $(\kappa=1,0)$ & 2312,04 & 2916,63 & 3666,34 & 2773,08 \\
$10 \times 20$ & Matérn $(\kappa=2,0)$ & 2311,98 & 2916,52 & 3666,33 & 2773,97 \\
& Exponencial & 4917,19 & 6002,84 & 7091,53 & 5835,47 \\
& Matérn $(\kappa=1,0)$ & 4916,88 & 6002,84 & 7090,87 & 5835,47 \\
$10 \times 30$ & Matérn $(\kappa=2,0)$ & 4916,65 & 6002,84 & 7090,42 & 5835,47 \\
& Exponencial & 2741,62 & 3445,61 & 4277,13 & 3337,81 \\
$10 \times 40$ & Matérn $(\kappa=1,0)$ & 2741,37 & 3445,61 & 4276,59 & 3337,76 \\
& Matérn $(\kappa=2,0)$ & 2741,19 & 3445,61 & 4276,42 & 3337,71 \\
& Exponencial & 2303,18 & 2927,16 & 3702,12 & 2768,40 \\
10 x 50 & Matérn $(\kappa=1,0)$ & 2302,82 & 2927,10 & 3701,94 & 2768,40 \\
& Matérn $(\kappa=2,0)$ & 2302,45 & 2927,06 & 3701,82 & 2768,40 \\
& Exponencial & 1583,94 & 2039,18 & 2620,19 & 1926,77 \\
& Matérn $(\kappa=1,0)$ & 1583,79 & 2039,18 & 2619,87 & 1926,77 \\
& Matérn $(\kappa=2,0)$ & 1583,73 & 2039,18 & 2619,61 & 1926,76 \\
& Exponencial & 2298,20 & 2912,17 & 3692,13 & 2765,27 \\
& Matérn $(\kappa=1,0)$ & 2298,12 & 2912,17 & 3692,23 & 2765,20 \\
& Matérn $(\kappa=2,0)$ & 2298,04 & 2912,17 & 3692,33 & 2765,15 \\
\hline
\end{tabular}

Tabela 6. Critério de informação de Akaike para o modelo espacial $\left(\mathrm{AIC}_{\mathrm{sp}}\right)$ e não-espacial $\left(\mathrm{AIC}_{\mathrm{nsp}}\right)$, diferenças dos $\operatorname{AIC}\left(\Delta_{\mathrm{sp}}\right)$ e efeito pepita relativo $\left(\operatorname{Rel}_{\tau^{2}}\right)$ das quatro formações florestais com diferentes tamanhos e formas de parcela para área basal $\left(\mathrm{m}^{2} \cdot \mathrm{ha}^{-1}\right)$.

Table 6. Akaike information criterion for spatial models $\left(\mathrm{AIC}_{\mathrm{sp}}\right)$ and not spatial $\left(\mathrm{AIC}_{\mathrm{nsp}}\right)$, AIC differences $\left(\Delta_{\mathrm{sp}}\right)$ and relative nugget $\left(\operatorname{Rel}_{\tau^{2}}\right)$ for four forest formations with different sizes and shapes of sampling units for basal area $\left(\mathrm{m}^{2} \cdot \mathrm{ha}^{-1}\right)$.

\begin{tabular}{|c|c|c|c|c|c|c|c|c|}
\hline Parcela (m) & $\mathrm{AIC}_{\mathrm{nsp}}$ & $\mathrm{AIC}_{\mathrm{sp}}$ & $\Delta_{\mathrm{sp}}$ & $\operatorname{Rel}_{\tau^{2}}$ & $\mathbf{A I C}_{\text {nsp }}$ & $\mathrm{AIC}_{\mathrm{sp}}$ & $\Delta_{\mathrm{sp}}$ & $\mathbf{R e l}_{\tau^{2}}$ \\
\hline & \multicolumn{4}{|c|}{ Savana } & \multicolumn{4}{|c|}{ Estacional } \\
\hline $5 \times 5$ & 32034,74 & 31985,05 & 49,69 & 0,97 & 28585,24 & 28561,02 & 24,22 & 0,71 \\
\hline $10 \times 10$ & 6977,62 & 6941,46 & 36,16 & 0,92 & 8446,28 & 8450,23 & $-3,96$ & 0,69 \\
\hline $20 \times 20$ & 1476,19 & 1454,58 & 21,61 & 0,77 & 1879,58 & 1883,24 & $-3,66$ & - \\
\hline $10 \times 20$ & 3215,78 & 3204,93 & 10,85 & 0,92 & 4033,82 & 4037,82 & $-4,00$ & 0,71 \\
\hline $10 \times 30$ & 1741,95 & 1733,14 & 8,80 & 0,91 & 2259,48 & 2263,48 & $-4,00$ & 0,73 \\
\hline $10 \times 40$ & 1455,34 & 1444,65 & 10,69 & 0,86 & 1898,37 & 1901,44 & $-3,06$ & - \\
\hline $10 \times 50$ & 989,32 & 982,72 & 6,59 & 0,86 & 1311,24 & 1315,24 & $-4,00$ & 0,72 \\
\hline $5 \times 80$ & 1472,59 & 1448,46 & 24,12 & 0,58 & 1875,87 & 1879,87 & $-4,00$ & 0,53 \\
\hline & \multicolumn{4}{|c|}{ Ombrófila } & \multicolumn{4}{|c|}{ Restinga } \\
\hline $5 \times 5$ & 23274,88 & 22390,99 & 783,89 & 0,75 & 33415,54 & 33413,64 & 1,90 & 0,97 \\
\hline $10 \times 10$ & 8701,16 & 8439,65 & 261,51 & 0,62 & 8438,05 & 8441,71 & $-3,66$ & - \\
\hline $20 \times 20$ & 2181,86 & 2097,84 & 84,01 & 0,41 & 1818,00 & 1816,98 & 1,03 & 0,93 \\
\hline $10 \times 20$ & 4453,50 & 4420,22 & 33,28 & 0,84 & 3941,47 & 3945,47 & $-4,00$ & 0,72 \\
\hline $10 \times 30$ & 2586,13 & 2557,76 & 28,37 & 0,60 & 2219,42 & 2223,05 & $-3,63$ & - \\
\hline $10 \times 40$ & 2165,91 & 2163,60 & 2,32 & 0,88 & 1808,71 & 1812,71 & $-4,00$ & 0,73 \\
\hline $10 \times 50$ & 1530,00 & 1527,61 & 2,39 & 0,80 & 1247,47 & 1251,47 & $-4,00$ & 0,70 \\
\hline $5 \times 80$ & 2150,78 & 2114,41 & 36,37 & 0,46 & 1803,37 & 1806,64 & $-3,27$ & 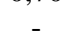 \\
\hline
\end{tabular}

FLORESTA, Curitiba, PR, v. 40, n. 4, p. 849-860, out./dez. 2010.

Oda-Souza, M. et al. 
Tabela 7. Critério de informação de Akaike para o modelo espacial $\left(\mathrm{AIC}_{\mathrm{sp}}\right)$ e não-espacial $\left(\mathrm{AIC}_{\mathrm{nsp}}\right)$, diferenças dos AIC $\left(\Delta_{\mathrm{sp}}\right)$ e efeito pepita relativo $\left(\operatorname{Rel}_{\tau^{2}}\right)$ das quatro formações florestais com diferentes tamanhos e formas de parcela para biomassa $\left(\mathrm{mg}^{-h^{-1}}\right)$.

Table 7. Akaike information criterion for spatial models $\left(\mathrm{AIC}_{\mathrm{sp}}\right)$ and not spatial $\left(\mathrm{AIC}_{\mathrm{nsp}}\right)$, AIC differences $\left(\Delta_{\mathrm{sp}}\right)$ and relative nugget $\left(\operatorname{Rel}_{\tau^{2}}\right)$ for four forest formations with different sizes and shapes of sampling units for biomass $\left(\mathrm{mg}^{-\mathrm{ha}^{-1}}\right)$.

\begin{tabular}{|c|c|c|c|c|c|c|c|c|}
\hline Parcela (m) & $\mathbf{A I C}_{\mathrm{nsp}}$ & $\mathrm{AIC}_{\mathrm{sp}}$ & $\Delta_{\mathrm{sp}}$ & $\operatorname{Rel}_{\tau^{2}}$ & $\mathbf{A I C}_{\mathrm{nsp}}$ & $\mathbf{A I C}_{\mathrm{sp}}$ & $\Delta_{\mathrm{sp}}$ & $\operatorname{Rel}_{\tau^{2}}$ \\
\hline & \multicolumn{4}{|c|}{ Savana } & \multicolumn{4}{|c|}{ Estacional } \\
\hline $5 \times 5$ & 45144,89 & 45112,92 & 31,98 & 0,98 & 39976,16 & 39961,60 & 14,56 & 0,77 \\
\hline $10 \times 10$ & 10372,64 & 10350,52 & 22,12 & 0,93 & 12132,24 & 12136,20 & $-3,95$ & 0,74 \\
\hline $20 \times 20$ & 2323,16 & 2312,04 & 11,12 & 0,82 & 2913,19 & 2916,63 & $-3,44$ & - \\
\hline $10 \times 20$ & 4916,66 & 4916,88 & $-0,22$ & 0,96 & 5998,84 & 6002,84 & $-4,00$ & 0,50 \\
\hline $10 \times 30$ & 2740,20 & 2741,37 & $-1,16$ & 0,97 & 3441,61 & 3445,61 & $-4,00$ & 0,74 \\
\hline $10 \times 40$ & 2303,00 & 2302,82 & 0,18 & 0,93 & 2924,05 & 2927,10 & $-3,05$ & - \\
\hline $10 \times 50$ & 1584,26 & 1583,79 & 0,46 & - & 2035,18 & 2039,18 & $-4,00$ & 0,71 \\
\hline \multirow{2}{*}{$5 \times 80$} & 2316,78 & 2298,12 & 18,66 & 0,59 & 2908,17 & 2912,17 & $-4,00$ & 0,52 \\
\hline & \multicolumn{4}{|c|}{ Ombrófila } & \multicolumn{4}{|c|}{ Restinga } \\
\hline $5 \times 5$ & 30362,76 & 29788,58 & 574,18 & 0,80 & 46619,87 & 46619,96 & $-0,07$ & 0,98 \\
\hline $10 \times 10$ & 12998,15 & 12859,10 & 139,05 & 0,76 & 12139,16 & 12142,83 & $-3,67$ & - \\
\hline $20 \times 20$ & 3697,66 & 3666,34 & 31,33 & 0,64 & 2777,29 & 2773,08 & 4,20 & 0,90 \\
\hline $10 \times 20$ & 7102,58 & 7090,87 & 11,72 & 0,92 & 5831,47 & 5835,47 & $-4,00$ & 0,73 \\
\hline $10 \times 30$ & 4287,63 & 4276,59 & 11,03 & 0,78 & 3336,41 & 3337,76 & $-1,34$ & 0,95 \\
\hline $10 \times 40$ & 3699,41 & 3701,94 & $-2,54$ & 0,96 & 2764,40 & 2768,40 & $-4,00$ & 0,71 \\
\hline $10 \times 50$ & 2617,23 & 2619,87 & $-2,64$ & 0,94 & 1922,84 & 1926,77 & $-3,92$ & - \\
\hline $5 \times 80$ & 3698,55 & 3692,23 & 6,32 & 0,82 & 2762,51 & 2765,20 & $-2,69$ & - \\
\hline
\end{tabular}

As estimativas dos parâmetros do modelo geoestatístico para as variáveis área basal e biomassa são apresentadas nas tabela 8 e 9, respectivamente. Em geral, pode-se observar que com o aumento do tamanho e mudança na forma da parcela as estimativas dos parâmetros patamar $\left(\sigma^{2}\right)$ e efeito pepita $\left(\tau^{2}\right)$ diminuiram nas quatro formações florestais estudadas. $\mathrm{O}$ efeito pepita relativo $\left(\operatorname{Rel}_{\tau^{2}}\right)$ diminuiu com o aumento do tamanho da parcela.

Os alcances práticos (a), que determinam a distância a partir da qual a variável não tem mais influência relevante sobre a vizinhança, foram maiores para as formações Savana e Ombrófila, e menores para Estacional e Restinga, exceto para parcelas de 20 × $20 \mathrm{~m}$.

Avaliando somente para as parcelas quadradas, as estimativas da variância estrutura $\left(\sigma^{2}\right)$, do componente aleatório $\left(\tau^{2}\right)$ e do efeito pepita relativo $\left(\operatorname{Rel}_{\tau^{2}}\right)$ diminuíram com o aumento do tamanho da parcela. Os menores valores de alcance prático (a) foram encontrados nas parcelas de $10 \times 10 \mathrm{~m}$ para as formações de Savana, Estacional e Restinga, e 5 x $5 \mathrm{~m}$ para Ombrófila. Nas parcelas de mesmo tamanho $\left(400 \mathrm{~m}^{2}\right)$ e diferentes formas para as variáveis área basal e biomassa, os valores das estimativas $\left(\sigma^{2}\right)$ foram menores, e do efeito pepita relativo $\left(\operatorname{Rel}_{\tau^{2}}\right)$ maiores, para as formações de Savana e Ombrófila, considerando parcelas de $10 \times 40 \mathrm{~m}$.

\section{DISCUSSÃO}

As mudança de tamanhos e formas de parcela têm um grande impacto na distribuição dos dados. Avaliando a simetria das distribuições das variáveis área basal e biomassa, pode-se observar que parcelas pequenas têm maior probabilidade de conter valores extremos, levando a maiores variâncias e distribuições assimétricas. $\mathrm{O}$ aumento do tamanho da parcela leva à diluição dos valores extremos, com o efeito de reduzir a dispersão e simetria dos dados, tornando as medidas de posição (média e mediana) semelhantes. Resultados similares também foram encontrados por Bellehumeur et al. (1997), em trabalho com densidade de plantas com uma parcela permanente de 50 ha na floresta tropical da Malásia, e por Rossi; Nuutinen (2004) analisando o efeito do tamanho da parcela na percepção do padrão espacial de minhocas. 
Tabela 8. Estimativas dos parâmetros $\left(\sigma^{2}, \tau^{2}\right)$, efeito pepita relativo $\left(\operatorname{Rel}_{\tau^{2}}\right)$ e alcance prático (a) das quatro formações florestais com diferentes tamanhos e formas de parcela para área basal $\left(\mathrm{m}^{2} \cdot \mathrm{ha}^{-1}\right)$.

Table 8. Estimates of model parameters $\left(\sigma^{2}, \tau^{2}\right)$, relative nugget $\left(\operatorname{Rel}_{\tau^{2}}\right)$ and practical range (a) of the four forest formations with different sizes and shapes of sampling units for basal area $\left(\mathrm{m}^{2} \cdot \mathrm{ha}^{-1}\right)$.

\begin{tabular}{|c|c|c|c|c|c|c|c|c|}
\hline Parcela (m) & $\sigma^{2}$ & $\tau^{2}$ & $\operatorname{Rel}_{\tau^{2}}$ & $\mathbf{a}$ & $\sigma^{2}$ & $\tau^{2}$ & $\operatorname{Rel}_{\tau^{2}}$ & $\mathbf{a}$ \\
\hline & \multicolumn{4}{|c|}{ Savana } & \multicolumn{4}{|c|}{ Estacional } \\
\hline $5 \times 5$ & 0,30 & 10,74 & 0,97 & 271,40 & 2,80 & 6,79 & 0,71 & 8,16 \\
\hline $10 \times 10$ & 0,22 & 2,39 & 0,92 & 183,73 & 0,66 & 1,50 & 0,69 & 7,39 \\
\hline $20 \times 20$ & 0,20 & 0,68 & 0,77 & 214,06 & 0,59 & 0,00 & - & 17,05 \\
\hline $10 \times 20$ & 0,12 & 1,35 & 0,92 & 179,86 & 0,33 & 0,83 & 0,71 & 2,52 \\
\hline $10 \times 30$ & 0,08 & 0,82 & 0,91 & 458,05 & 0,20 & 0,54 & 0,73 & 2,28 \\
\hline $10 \times 40$ & 0,11 & 0,68 & 0,86 & 212,18 & 0,64 & 0,00 & - & 10,65 \\
\hline $10 \times 50$ & 0,09 & 0,56 & 0,86 & 306,26 & 0,15 & 0,40 & 0,72 & 2,46 \\
\hline \multirow[t]{2}{*}{$5 \times 80$} & 0,36 & 0,48 & 0,58 & 27,83 & 0,27 & 0,31 & 0,53 & 0,08 \\
\hline & \multicolumn{4}{|c|}{ Ombrófila } & \multicolumn{4}{|c|}{ Restinga } \\
\hline $5 \times 5$ & 3,82 & 11,38 & 0,75 & 70,53 & 0,26 & 7,70 & 0,97 & 19,70 \\
\hline $10 \times 10$ & 2,41 & 4,01 & 0,62 & 91,49 & 1,86 & 0,00 & - & 7,17 \\
\hline $20 \times 20$ & 1,58 & 1,09 & 0,41 & 101,73 & 0,03 & 0,44 & 0,93 & 237,04 \\
\hline $10 \times 20$ & 0,66 & 3,51 & 0,84 & 241,02 & 0,25 & 0,67 & 0,72 & 2,32 \\
\hline $10 \times 30$ & 1,73 & 2,55 & 0,60 & 473,63 & 0,66 & 0,00 & - & 9,15 \\
\hline $10 \times 40$ & 0,31 & 2,29 & 0,88 & 406,26 & 0,13 & 0,33 & 0,73 & 2,41 \\
\hline $10 \times 50$ & 0,57 & 2,25 & 0,80 & 466,71 & 0,12 & 0,28 & 0,70 & 2,66 \\
\hline $5 \times 80$ & 1,22 & 1,04 & 0,46 & 25,69 & 0,45 & 0,00 & - & 5,13 \\
\hline
\end{tabular}

Tabela 9. Estimativas dos parâmetros $\left(\sigma^{2}, \tau^{2}\right)$, efeito pepita relativo $\left(\operatorname{Rel}_{\tau^{2}}\right)$ e alcance prático (a) das quatro formações florestais com diferentes tamanhos e formas de parcela para biomassa (mg.ha $\left.{ }^{-1}\right)$.

Table 9. Estimates of model parameters $\left(\sigma^{2}, \tau^{2}\right)$, relative nugget $\left(\operatorname{Rel}_{\tau^{2}}\right)$ and pratical range (a) of the four forest formations with different sizes and shapes of sampling units for biomass $\left(\mathrm{mg}^{2} \mathrm{ha}^{-1}\right)$.

\begin{tabular}{|c|c|c|c|c|c|c|c|c|}
\hline Parcela (m) & $\sigma^{2}$ & $\tau^{2}$ & $\operatorname{Rel}_{\tau^{2}}$ & $\mathbf{a}$ & $\sigma^{2}$ & $\tau^{2}$ & $\operatorname{Rel}_{\tau^{2}}$ & $\mathbf{a}$ \\
\hline & \multicolumn{4}{|c|}{ Savana } & \multicolumn{4}{|c|}{ Estacional } \\
\hline $5 \times 5$ & 1,25 & 60,65 & 0,98 & 230,83 & 4,83 & 16,46 & 0,77 & 8,32 \\
\hline $10 \times 10$ & 1,06 & 14,39 & 0,93 & 149,65 & 1,73 & 4,97 & 0,74 & 7,48 \\
\hline $20 \times 20$ & 0,90 & 4,16 & 0,82 & 187,10 & 2,30 & 0,00 & - & 18,30 \\
\hline $10 \times 20$ & 0,33 & 8,30 & 0,96 & 148,86 & 2,05 & 2,02 & 0,50 & 2,52 \\
\hline $10 \times 30$ & 0,18 & 5,09 & 0,97 & 283,64 & 0,72 & 2,01 & 0,74 & 2,38 \\
\hline $10 \times 40$ & 0,33 & 4,24 & 0,93 & 164,39 & 2,45 & 0,00 & - & 10,69 \\
\hline $10 \times 50$ & 3,71 & 0,00 & - & 15,00 & 0,60 & 1,51 & 0,71 & 2,41 \\
\hline \multirow[t]{2}{*}{$5 \times 80$} & 1,96 & 2,86 & 0,59 & 24,87 & 1,07 & 1,18 & 0,52 & 0,08 \\
\hline & \multicolumn{4}{|c|}{ Ombrófila } & \multicolumn{4}{|c|}{ Restinga } \\
\hline $5 \times 5$ & 6,75 & 27,84 & 0,80 & 76,53 & 0,52 & 20,76 & 0,98 & 21,13 \\
\hline $10 \times 10$ & 5,33 & 16,99 & 0,76 & 97,28 & 5,75 & 0,00 & - & 7,18 \\
\hline $20 \times 20$ & 4,35 & 7,71 & 0,64 & 94,98 & 0,16 & 1,44 & 0,90 & 294,80 \\
\hline $10 \times 20$ & 1,36 & 15,72 & 0,92 & 243,73 & 0,81 & 2,16 & 0,73 & 2,53 \\
\hline $10 \times 30$ & 3,37 & 12,13 & 0,78 & 471,36 & 0,11 & 2,06 & 0,95 & 161,53 \\
\hline $10 \times 40$ & 0,41 & 10,94 & 0,96 & 475,99 & 0,42 & 1,07 & 0,71 & 2,57 \\
\hline $10 \times 50$ & 0,69 & 10,62 & 0,94 & 558,18 & 1,31 & 0,00 & - & 8,20 \\
\hline $5 \times 80$ & 2,01 & 9,32 & 0,82 & 58,05 & 1,48 & 0,00 & - & 5,60 \\
\hline
\end{tabular}


A função de correlação utilizada, Matérn, foi adotada devido à flexibilidade que ela oferece para modelar o decaimento da correlação, bem como a suavidade do processo, através da modificação de seus parâmetros. A suavidade do processo $\mathrm{S}(\mathrm{x})$ é estabelecida pelos parâmetros de alcance $(\phi)$ e $\kappa$ (BOGNOLA et al., 2008). A utilização de diferentes $\kappa(\kappa=0,5,1,0$ e 2,0) na modelagem das formações permitiu que variações suaves ou abruptas como, por exemplo, as parcelas de 5 × $5 \mathrm{~m}$ na formação Ombrófila, fossem detectadas. Uma possível explicação para essa variação observada na formação Ombrófila, talvez, seja a topografia altamente acidentada, a presença de clareiras e grandes quantidades de bambus, captada em variações de pequena escala e filtrada com o aumento do tamanho da parcela.

Capretz (2004), utilizando o mesmo conjunto de dados da presente pesquisa e analisando o padrão espacial da distribuição das árvores pela função K de Ripley, concluiu que as quatro formações florestais apresentam padrões espaciais distintos e em diferentes escalas. A formação Ombrófila mostrou um padrão mais complexo de agregação, diferenciando-se das demais formações. No entanto foram encontradas semelhanças entre o padrão espacial da Savana e da Restinga, e o padrão espacial foi predominamente aleatório para a formação Estacional. No presente trabalho, a estrutura de continuidade espacial da formação Ombrófila também se mostrou complexa e mais pronunciada, seguida pela Savana, e relativamente fracas para as formações Estacional e Restinga.

Fortin (1999), trabalhando com diferentes tamanhos e formas de parcela para densidade de três espécies de árvores com o coeficiente de autocorrelação espacial de Moran's I, verificou que a magnitude da estrutura espacial aumentou com o aumento do tamanho da parcela para as parcelas de $10 \mathrm{x} 20 \mathrm{~m}$, $20 \times 10 \mathrm{~m}$ e $15 \times 15 \mathrm{~m}$, e diminuiu com parcelas de $20 \times 20 \mathrm{~m}$. Segundo esse autor, isso pode ser explicado pelo fato de que parcelas maiores incluem maiores variabilidades, que resultam em menor autocorrelação espacial.

Resultados contrários foram encontrados em estudos baseados em variogramas Bellehumeur et al. (1997). Esses autores, trabalhando com parcelas quadradas, verificaram que o processo não se apresenta espacialmente estruturado nas parcelas de $5 \times 5 \mathrm{~m}$, contudo é fortemente estruturado nas parcelas de $20 \times 20 \mathrm{~m}$. Neste estudo, as parcelas quadradas foram fortemente estruturadas para as formações de Savana e Ombrófila, mesmo para as parcelas de 5 x $5 \mathrm{~m}$, que apresentaram baixa proporção da variância estruturada. Oliveira (2003) argumenta que, embora demande uma metodologia mais complexa e maior tempo computacional, a obtenção das estimativas dos parâmetros baseada em modelos e as interpretações dos resultados são elaboradas com maior confiabilidade quando comparados aos métodos em que as estimativas dos parâmetros são obtidas através do semivariograma de forma subjetiva.

Nas formações Estacional e Restinga, as mudanças do tamanho e forma das parcelas não influenciaram a estrutura de dependência. Por outro lado, nas formações Savana e Ombrófila essa dependência foi intensificada com o aumento do tamanho da parcela quadrada e não detectada para algumas parcelas com forma retangular para a variável biomassa. Para essas áreas, as parcelas quadradas com dimensões de 20 × 20 m captaram a estrutura de dependência espacial, e as retangulares, 10 × 40 m, captaram a variabilidade das quatro formações, não detectando a estrutura continuidade espacial.

Fortin (1999) não recomenda o uso de parcelas retangulares quando o objetivo do estudo é detectar a estrutura de continuidade espacial, pois a magnitude da autocorrelação espacial pode ser sub ou superestimada em função de a orientação da parcela corresponder, ou não, aos padrões espaciais da população em estudo.

Apesar de a dependência espacial da formação Ombrófila ser mais pronunciada do que a da Savana, foi observado que a estrutura de continuidade espacial da Savana se apresentou em escala mais ampla do que a estrutura da Ombrófila para a variável área basal. Enquanto que para a Ombrófila a parcela com 10 x $40 \mathrm{~m}$ apresentou o modelo espacial no limiar do valor proposto para plausibilidade do modelo geoestatístico, na Savana Florestada o modelo espacial foi consideravelmente mais plausível que o modelo não-espacial. Isso mostra que na Ombrófila as parcelas retangulares captaram o padrão espacial da floresta conforme sugerido por Fortin (1999).

\section{CONCLUSÕES}

- Devido à distinção da estrutura de dependência espacial e de suas escalas de ocorrência, foi verificado que não há como determinar um tamanho e forma ótimos da unidade amostral para as quatro formações florestais estudadas. Estes dependerão das características de cada formação e dos vários processos ecológicos que podem estar atuando de forma simultânea na definição da estrutura espacial dessas formações. 
- É clara a influência do tamanho e forma da parcela sobre a percepção da estrutura de continuidade espacial. Quando estudos sobre essa estrutura não forem possíveis, parcelas de forma retangular são preferidas, pois são capazes de captar a variabilidade das quatro formações. Porém, a não detecção da estrutura espacial não significa que ela inexista, pois quando ela é detectada por algum tamanho e forma de parcela, é inerente ao processo. No caso de trabalhos que objetivam a detecção da estrutura espacial e predição, parcelas de 20 × 20 m descrevem melhor a estrutura de continuidade espacial.

\section{AGRADECIMENTOS}

À CAPES, pelo apoio financeiro com a manutenção da bolsa de auxílio. À FAPESP, ao Projeto "Biota Parcela Permanentes", pela concessão dos dados para a realização deste trabalho.

\section{REFERÊNCIAS}

AKAIKE, H. A New look at statistical model identification. Transactions on Automatic Control. New York, v. 19, n. 6, p. 717-723, 1974.

BELLEHUMEUR, C ; LEGENDRE, P. ; MARCOTTE, D. Variance and spatial scales in a tropical rain forest: changing the size of sampling units. Plant Ecology. Belgium, v. 130, p. 89-98, 1997.

BELLEHUMEUR, C ; LEGENDRE, P. Multiscale sources of variation in ecological variables: modeling spatial dispersion, elaborating sampling designs. Landscape Ecology, Netherlands, v. 13, p. 15-25, 1998.

BOGNOLA, I. A.; RIBEIRO JR., P. J.; SILVA, E.A.A.; LINGNAU, C.; HIGA, A. R. Modelagem uni e bivariada da variabilidade espacial de rendimento de Pinus taeda L. Floresta, Curitiba, v. 38, n. 2, p. 373$385,2008$.

BOX, G. E. P.; COX, D. R. An analysis of transformations. Journal of the Royal Statistical Society, London, v. 26, n. 2, p. 211-252, 1964.

BURGER, D. M. Modelos alométricos para a estimativa da fitomassa de Mata Atlântica na Serra do Mar, SP. São Paulo, 2005. 112 p. Tese (Doutorado) - Instituto de Biociências, Universidade de São Paulo.

BURNHAM, K. P.; ANDERSON, D. R. Model selection and multimodel inference. 2. ed. New York: Springer, 2002. $350 \mathrm{p}$.

CAPRETZ, R. L. Análise de padrões espaciais de árvores em quatro formações florestais do estado de São Paulo, através de análises de segunda ordem, com a função K de Ripley. Piracicaba, 2004. 93 p. Dissertação (Mestrado) - Escola Superior de Agricultura "Luiz de Queiroz", Universidade de São Paulo.

CHEN, Y. F.; YU, F. H.; DONG, M. Scale-dependent spatial heterogeneity of vegetation in Mu Us sandy land, a semi-arid area of China. Plant Ecology, Netherlands, v. 162, p. 135-142, 2002.

CHRISTENSEN, O. F.; DIGGLE, P. J.; RIBEIRO JR., P. J. Analysing positive-valued spatial data: the transformed gaussian model. In geoENV - Geostatistics for Environmental Applications. P. Monestiez, D. Allard and R. Froidevaux, Eds., Kluwer, p. 287-298, Amsterdam, 2001.

DIGGLE, P. J.; TAWN, J. A.; MOYEED, R. A. Model-Based geostatistics. Applied Statistics, London, v. 47, p. 299-350, 1998.

DIGGLE, P. J.; RIBEIRO JR., P. J. Model-Based geostatistics. New York: Springer, 228 p., 2007.

DORMANN, C. F. Effects of incorporating spatial autocorrelation into the analysis of species distribution data. Global Ecology and Biogeography, Oxford, v. 16, p. 129-138, 2007.

DUNGAN, J. L.; PERRY, J. N.; DALE, M. R. T.; LEGENDRE, P.; CITRON-POUSTY, S.; FORTIN, JAKOMULSKA, M. J.; MIRITI, M.; ROSENBERG, M. S. A balanced view of scale in spatial statistical analysis. Ecography, Copenhagen, v. 25, p. 626-640, 2002. 
DUTILLEUL, P. Spatial heterogeneity and the design of ecological field experiments. Ecology, Tempe, v. 74, n. 6., p. 1646-1658, 1993.

FORTIN, M. J. Effects of sampling unit resolution on the estimation of spatial autocorrelation. Ecoscience, Saint-Foy, v. 6, p. 636-641, 1999.

GOSLEE, S. C. Behavior of vegetation sampling methods in the presence of spatial autocorrelation. Plant Ecology, Dordrecht, n. 187, p. 203-212, 2006.

GOTWAY, C. A.; YOUNG, L. J. Combining incompatible spatial data. Journal of the American Statistical Association, New York, v. 97, p. 632-648, 2002.

HE, F.; LaFRANKIE, J. V.; SONG, B. Scale dependence of tree abundance and richness in a tropical rain forest. Landscape Ecology, Netherlands, v. 17, p. 559-568, 2002.

HERNANDEZ-STEFANONI, J. L. Relationships between landscape patterns and species richness of trees, shrubs and vines in a tropical forest Plant Ecology, Belgium, v. 179, p. 53-65, 2005.

HOLMES, K. W.; CHADWICK, O. A.; KYRIAKIDIS, P. C.; FILHO, E. P. S. de; SOARES, J. V.; ROBERTS, D. A. Large-area spatially explicit estimates of tropical soil carbon stocks and response to land-cover change. Global Biogeochemical Cycles, Washington, v. 20, p. 1-12, 2006.

ISAAKS, E. H.; SRIVASTAVA, R. H. Applied Geostatisctics. New York: Oxford University Press, 1989. $561 \mathrm{p}$.

KULLBACK, S.; LEIBLER, R. A. On information and sufficiency. Annals of Mathematical Statistics, Ann Arbor, v. 22, p. 79-86, 1951.

LEGENDRE, P.; FORTIN, M. J. Spatial pattern and ecological analysis. Vegetatio, Belgium, v. 80, p. 107-138, 1989.

OLIVEIRA, M. C. N. Métodos de estimação de parâmetros em modelos geoestatísticos com diferentes estruturas de covariância: uma aplicação ao teor de cálcio no solo. Piracicaba, 2003. 140 p. Tese (Doutorado) - Escola Superior de Agricultura "Luiz de Queiroz", Universidade de São Paulo.

PLONER, A.; DUTTER, R. New directions in geostatistics. Journal of Statistical Planning and Inference, Amsterdam, v. 91, p. 499-509, 2000.

R DEVELOPMENT CORE TEAM. R: A language and environment for statistical computing. $R$ Foundation for Statistical Computing, Vienna, Austria. ISBN 3-900051-07-0, http://www.R-project.org , 2008.

RIBEIRO JR., P. J.; DIGGLE, P. J. The geoR package functions for geostatistical data analysis. RNEWS, Vienna, v. 1, p. 15-18, 2001.

RODRIGUES, R. R. Relatório temático do projeto parcelas permanentes. Laboratório de Ecologia e Restauração Florestal, ESALQ/USP. Piracicaba, 2002. Disponível em: <http://www.lerf.esalq.usp.br/ parcelas/relatorio1.pdf>. Acesso em 4 fev. 2009.

OSSI, J. P.; NUUTINEN, V. The effect of sampling unit size on the perception of the spatial pattern of earthworm (Lumbricus terrestris L.) middens. Applied Soil Ecology, Amsterdam, v. 27, p. 189-196, 2004.

ROSSI, R. E.; MULLA, D. J.; JOURNEL, A. G.; FRANZ, E. H. Geostatistical tools for modeling and interpreting ecological spacial dependence. Ecological Monographs, Lawrence, v. 62, n. 2, p. 277-341, 1992.

SALES, M. H.; SOUZA JR., C. M.; KYRIAKIDISB, P. C.; ROBERTS, Dar A.; VIDAL, E. Improving spatial distribution estimation of forest biomass with geostatistics: a case study for Rondônia, Brazil. Ecological Modelling, Amsterdam, n. 205, p. 221-230, 2007.

SATAKE, A.; IWASA, Y. Spatially limited pollen exchange and a long-range synchronization of trees. Ecology, Tempe, v. 83, p. 993-1005, 2002. 